DOI: http://dx.doi.org/10.35381/r.k.v4i8.265

\title{
La conciencia fonológica: Análisis en niños de educación inicial de instituciones peruanas según su estructura familiar
}

The phonological awareness: Analysis in children of initial education of peruvian institutions according to their family structure

\author{
Fanny Miriam Sanabria Boudri \\ fanny miriam@hotmail.com \\ Universidad Nacional Enrique Guzmán y Valle \\ Perú \\ https://orcid.org/0000-0002-2462-2715
}

\author{
Félix José Colina Ysea \\ fcolina@universidadsise.edu.pe \\ Universidad SISE \\ Perú \\ https://orcid.org/0000-0002-6651-3509 \\ José Luis Albites Sanabria \\ jose.albsab@gmail.com \\ Universidad Nacional de Ingeniería \\ Universidad SISE \\ Perú \\ https://orcid.org/0000-0001-7688-6221
}

Recibido: 12 de enero del 2019

Aprobado: 01 de abril del 2019

\section{RESUMEN}

El presente estudio pretende determinar la diferencia existente a nivel de conciencia fonológica según la estructura familiar que presentan niños de nivel básico de instituciones peruanas. El estudio corresponde a un diseño no experimental descriptivo comparativo. La muestra fue determinada según criterio no probabilístico intencional, estuvo conformada por 200 estudiantes con edades entre 4, 5 y 6 años de educación inicial, de ambos sexos. La conciencia fonológica fue evaluada a través del Test de Habilidades Metalingüísticas (THM). Los resultados ofrecen evidencia 
que existen diferencias significativas en las habilidades metalingüísticas: segmentación silábica y detección de rimas en los niños de diferentes estructuras familiares. En género, las niñas obtuvieron una diferencia favorable frente a los niños varones. Además, se evidenció diferencia a favor de los niños provenientes de instituciones privadas. Por último, se evidenció diferencias significativas favorables en niños cinco y seis años de edad.

Descriptores:: conciencia fonológica; estructura familiar; monoparental; extensiva y nuclear.

\section{ABSTRACT}

The study aims to determine whether or not there is a significant difference in phonological awareness skills among Peruvian children with different family structures. The study corresponds to a comparative descriptive non-experimental design. The sample was selected by intentional non-probabilistic approach, it consisted of 200 students aged 4, 5 and 6 years of initial education, of both sexes. Phonological awareness was measured by metalinguistic Skills Test (THM). The results indicate that there are significant differences in metalinguistic abilities: syllabic segmentation and detection of rhymes by family structure. By genre, the difference was in favor of female children. By school type, the difference was in favor of children from private institutions. Last, there was a significant difference in favor of children of five and six years old.

Descriptors: phonological awareness, family structure, single parent, extended and nuclear.

\section{INTRODUCCIÓN}

Los factores asociados al aprendizaje infantil han despertado un gran interés en estas últimas décadas, tanto a las autoridades como a los maestros, quienes presentan distintas miradas con distintos planteamientos. Por un lado, está el tema de aprendizaje de la lectura y la producción de textos, la lectura está asociada al desarrollo del lenguaje oral; esto indica la importancia de la conciencia fonológica, permitiendo interiorizar en los niños, la relación que existe de las letras y sus sonidos. Así mismo, el informe de evaluación internacional de los logros educativos realizados a través de la prueba PISA (2009), señala que los estudiantes tienen serias 
dificultades para emplear la lectura como una herramienta eficaz en su desarrollo integral, limitando la obtención de conocimientos, destrezas y habilidades al momento de enfrentarse a un texto. Por lo tanto, la presente investigación será relevante por cuanto permitió en primer lugar conocer el nivel de conciencia fonológica de los estudiantes de Instituciones Públicas y Privadas.

\section{DESARROLLO}

\section{Situación problemática de la Educación en el Perú}

La educación en el Perú, es uno de los grandes problemas por superar en el área de la lectoescritura, pues el informe emitido por la Dirección de Educación de Lima Metropolitana (DREL: 2013), expresa "que de 20 alumnos del segundo grado de primaria, solo dos entienden lo que leen, o que del $26 \%$ de los alumnos del segundo de primaria, que en la evaluación censal comprendían lo que leían, ha descendido en el 2011 a un 15\%". Al respecto se puede afirmar que los estudiantes muestran deficiencias severas en la adquisición de los aprendizajes básicos: lectura, escritura, comprensión lectora y matemáticas; situación preocupante que se acentúa en la educación superior debido a la complejidad de los procesos de aprendizaje que este nivel demanda (MINEDU, 2001).

Las dificultades que presentan los estudiantes en el aprendizaje de la lectura y la escritura es multivariada, pues carecen del desarrollo de ciertas habilidades, como es la conciencia fonológica, sino también del poco apoyo del maestro o padres de familia como acción mediadora que le permitan superar estas dificultades.

Además, múltiples investigaciones realizadas en Europa, Estados Unido, y Latinoamérica, confirman que en edad escolar las habilidades directamente relacionadas con la lectura tienen que ver con las funciones lingüísticas, metalingüísticas, memoria verbal, la conciencia fonológica, los niveles semánticos y sintácticos del lenguaje oral. (Velarde, E.; Canales, R.; Melendez, M. y Lingan, S., 2013). 
En tal sentido, la conciencia fonológica es el mejor predictor del aprendizaje de la lectura, pues su desarrollo se inicia en la edad preescolar sin dejar de lado el entorno familiar, específicamente la composición o estructura familiar o las instituciones educativas tanto públicas o privadas, entre otros, que son variables importantes en el rendimiento de los niños en la lectura, pues existe una fuerte relación entre el ambiente alfabetizador familiar y el rendimiento de los niños en la lectura. (Jiménez, 2012).

En tal sentido, el entorno familiar es, por lo tanto, una condición imprescindible pero no fundamental en el adecuado desarrollo de la conciencia fonológica, las diferencias lingüísticas entre personas de la misma edad responden, a causas genéticas y a los hábitos lingüísticos familiares. La falta de un ambiente normal y estable en lo afectivo, puede constituir la causa de un retraso lingüístico no siempre recuperable.

Del mismo modo un entorno positivo, con padres que atienden a los hijos en tempranas edades con actividades como leerles cuentos, proponerles escuchar una lectura, les estimulan e influye positivamente en el desarrollo lingüístico, pues diversos estudios demuestran la influencia de los padres y el ambiente familiar sobre la adquisición y desarrollo de la habilidad lectora (Jiménez,2012).

Asimismo, considerando el tipo de instituciones, las instituciones privadas tenían mejores resultados que las escuelas públicas, pudiendo esto relacionarse a otras variables como los recursos socioeconómicos, las características geográficas, desempeño de los maestros, entre otros. (Velarde, E.2009). Es así, que los resultados en la última evaluación Censal de Estudiantes las instituciones públicas y privadas en matemática ambos presentan el mismo desempeño, mientras que en comprensión lectora la brecha se ha recortado (MINEDU, 2014).

En base a los antecedentes expresados, el objetivo del presente estudio yace en determinar la diferencia de la conciencia fonológica en los estudiantes de educación inicial de instituciones educativas públicas y privadas según su estructura famiiar. 


\section{METODOLOGÍA}

La presente investigación corresponde al diseño no experimental. A su vez se subdivide en diseño transversal descriptivo, Carrasco (2009) afirma. "Estos diseños se emplean para analizar y conocer las características, rasgos, propiedades y cualidades de un hecho o fenómeno de la realidad en un momento determinado del tiempo" (p 72).

\section{Población y Unidad de Análisis}

La unidad de análisis de esta investigación son los estudiantes de 4, 5 y 6 años que cursan Educación Inicial de Instituciones Públicas y Particulares pertenecientes a la UGEL 06. La población objeto de estudio son los estudiantes de educación inicial de instituciones peruanas de la UGEL 06.

\section{Tamaño de muestra}

La muestra estuvo conformada por 200 estudiantes, distribuidos de la siguiente manera.

Tabla 1. Distribución de la muestra según tipo de institución

\begin{tabular}{lcc}
\hline Institución educativa & F & $\%$ \\
\hline Público & 93 & $47 \%$ \\
Particular & 107 & $53 \%$ \\
Total & 200 & $100 \%$ \\
\hline
\end{tabular}

Fuente: elaboración propia

En la tabla 1 se observa, que del $100 \%$ de los estudiantes, el $46,5 \%$ (93) pertenecen a instituciones educativas públicas y el 53,5\% (107) pertenecen a instituciones educativas particulares. 


\section{Criterios para la selección de muestra}

Para la selección de la muestra se utilizó el muestreo no probabilístico intencional. Al respecto Mata (1997) manifiesta que dicho muestreo "consiste en un conjunto de reglas, procedimientos y criterios mediante los cuales se selecciona un conjunto de elementos de una población que representan lo que sucede en toda esa población" ( $p$ 76). En tal sentido los criterios que se tomó en cuenta para la selección de la muestra fueron: Pertenencia a una Institución Educativa Estatal y Particular de la UGEL 06. Pertenecer a las aulas de inicial de 4, 5 y 6 años de edad.

\section{Instrumentos de recolección de datos.}

Para la recolección de datos se hizo uso del test de Habilidades Metalingüísticas (THM)

\section{Ficha Técnica}

Autores : Gómez, Valero, Buades y Pérez

Año : 1995

Rango de aplicación : Pre-Escolares

Administración : Individual

Descripción : El test de habilidades metalingüísticas (THM) está compuesto por siete sub test, que evalúa la conciencia fonológica, es decir, solo la metafonología, cuyos ítems se distribuyen de la siguiente forma:

\begin{tabular}{|l|c|}
\hline Sub Test & $\mathbf{N}^{\circ}$ de Items \\
\hline Segmentación Silábica & 20 \\
\hline Supresión Silábica & 12 \\
\hline Detección de Rimas & 12 \\
\hline Adiciones Silábicas & 10 \\
\hline Aislamiento de Fonemas & 8 \\
\hline
\end{tabular}




\begin{tabular}{|l|c|}
\hline Unión de Fonemas & 20 \\
\hline Conteo de Fonemas & 20 \\
\hline Total & 102 \\
\hline
\end{tabular}

Las puntuaciones de todas las sub pruebas oscilan entre 0 y 1 , siendo la máxima puntuación que puede obtener el examinado 7 y la mínima 0 . Cada ítem correctamente resuelto por el niño (a) se valora como 1 punto. La puntuación de cada sub test se obtiene hallando el cociente entre el número de aciertos del examinado y el número total de ítems del sub test. La puntuación total de la prueba es la suma de todos los cocientes de los sub test. De acuerdo a los resultados totales obtenidos se agrupan en 4 categorías:

Tabla 2. Baremo del instrumento

\begin{tabular}{|c|c|c|}
\hline Puntaje & Estadios & Descripción \\
\hline De 0 a 1, 75 & Deficiente & $\begin{array}{l}\text { Los alumnos acrecen de las habilidades } \\
\text { fonológicas básicas que facilitan el acceso a } \\
\text { la lectoescritura. }\end{array}$ \\
\hline $\begin{array}{l}\text { De } 1,75 \text { a } 3, \\
50\end{array}$ & Regular & $\begin{array}{l}\text { Alumnos capaces de desenvolverse con } \\
\text { dificultades en las sub pruebas } 1 \text { y } 3 \text {, pero } \\
\text { con dificultades manifiestas para operar con } \\
\text { eslabones silábicos, así como para } \\
\text { identificar palabras con premisas fonémicas } \\
\text { determinadas. }\end{array}$ \\
\hline $\begin{array}{l}\text { De } 3,50 \text { a } 5, \\
25\end{array}$ & Satisfactorio & $\begin{array}{l}\text { Alumnos que puntúan consistentemente en } \\
\text { los cinco primeros sub test de THM. } \\
\text { Fracasan, sin embargo con respecto a las } \\
\text { exigencias que plantean las sub pruebas } 6 \text { y }\end{array}$ \\
\hline
\end{tabular}


7.

De 5,25 a 7
Sobresalient Alumnos con un comportamiento fonológico

e brillante en todas las partes de la prueba.

\section{Confiabilidad del instrumento}

Este Test valora el grado de desarrollo de las habilidades metalingüísticas al iniciar el aprendizaje de la lectura. El presente estudio utilizó la adaptación hecha por Noemí Panca en el año 2000. La confiabilidad de esta adaptación se realizó mediante la técnica de dos mitades usando la fórmula de Kuder - Richardson 20 obteniéndose el puntaje de 0.81 y con la técnica de consistencia interna utilizando Alpha de Cronbach se obtuvo el puntaje de 0.81 . Se realizó la validez de contenido mediante el criterio de jueces y la validez de construcción por el método de análisis factorial exploratorio siendo la variante explicada 47.69. Asimismo, la medida de adecuación de muestreo de Kayser - Myer - Olking es de 0.83 .

\section{RESULTADOS}

En la tabla 3 se observa, que el mayor porcentaje de estudiantes que pertenecen al tipo familia monoparental carecen de habilidades fonológicas básicas, y los estudiantes que pertenecen al tipo de familia extensa; son capaces de desenvolverse con dificultades en las sub pruebas 1 y 3 , pero con dificultades manifiestas para operar con eslabones silábicos, así como para identificar palabras con premisas fonémicas determinadas. $Y$ los estudiantes que pertenecen a la familia nuclear sobresalen en los cinco primeros sub test del THM y fracasa en las sub pruebas 6 y 7 . Tabla 3. Diferencia de los niveles de conciencia fonológica según tipo de familia

\begin{tabular}{|c|c|c|c|c|c|}
\hline Niveles de & Monoparental & & & & \\
\hline $\begin{array}{l}\text { conciencia } \\
\text { fonolóaica }\end{array}$ & $\%$ & $F$ & $\%$ & $f$ & $\%$ \\
\hline
\end{tabular}


KOINONIA. Revista Arbitrada Interdisciplinaria de Ciencias de la Educación, Turismo, Ciencias Sociales y Económica, Ciencias del Agro y Mar y Ciencias Exactas y aplicadas. Año IV. Vol IV. N8. Julio - Diciembre 2019. Hecho el depósito de Ley: FA2016000010

\begin{tabular}{|c|c|c|c|c|c|c|}
\hline \multirow[t]{2}{*}{ Deficiente } & 13 & & 31 & $28 \%$ & 3 & \\
\hline & & $52 \%$ & & & & $12 \%$ \\
\hline Regular & 6 & $24 \%$ & 53 & $48 \%$ & 11 & $17 \%$ \\
\hline Satisfactorio & 4 & $16 \%$ & 15 & $14 \%$ & 28 & $44 \%$ \\
\hline Sobresaliente & 2 & $8 \%$ & 12 & $11 \%$ & 22 & $34 \%$ \\
\hline Total & & & & & & $100 \%$ \\
\hline 111 & $100 \%$ & 64 & & $0 \%$ & & \\
\hline
\end{tabular}

Fuente: elaboración propia

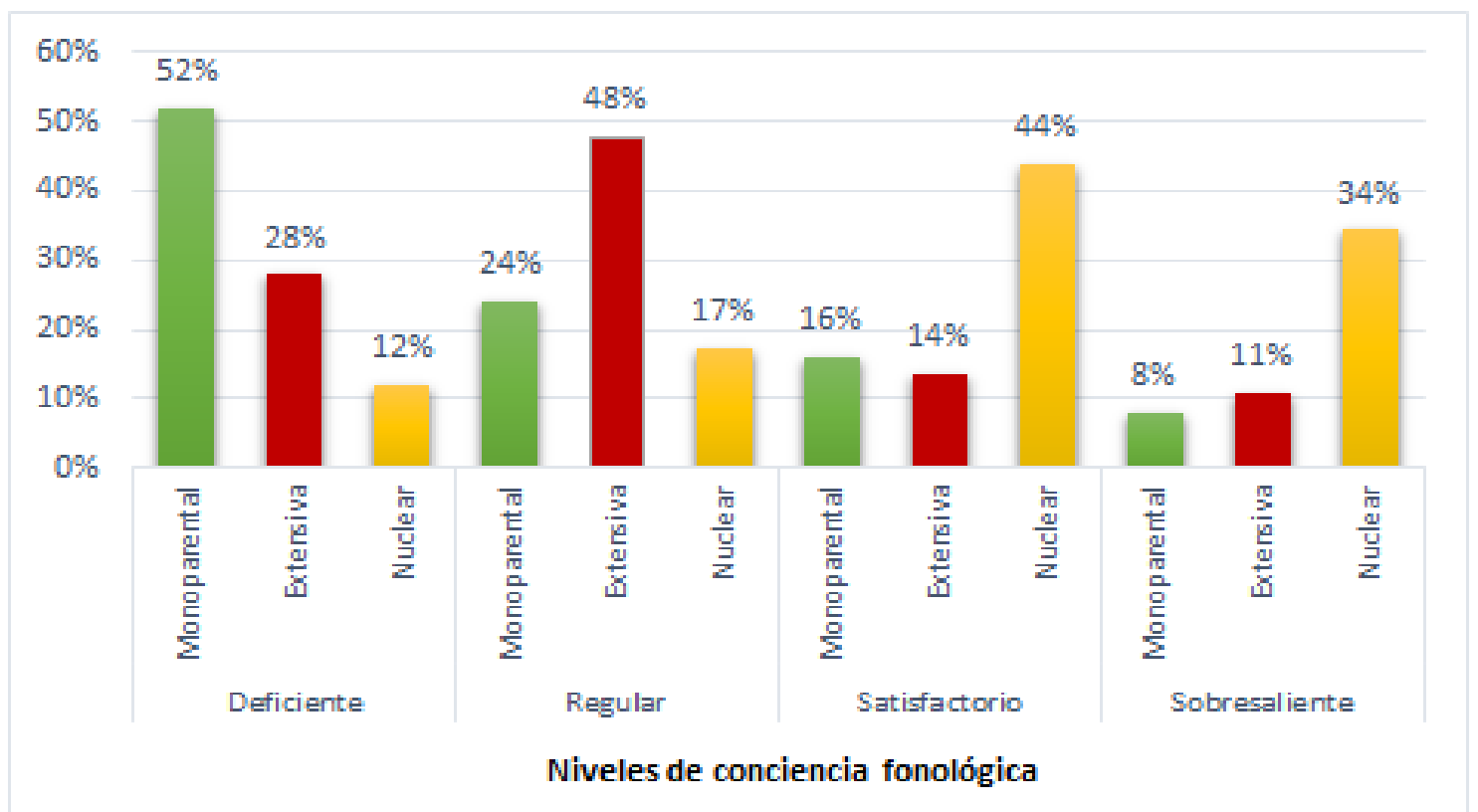

Fuente: elaboración propia

Figura 1. Distribución de porcentajes de los niveles de conciencia fonológica según tipo de familia en estudiantes de instituciones educativas públicas y privadas

\section{Niveles de conciencia fonológica según tipo de instituciones educativas}

En la tabla 4 y figura 2 se observa, que el mayor porcentaje de los estudiantes de las instituciones públicas; son capaces de desenvolverse con éxito en las sub pruebas 1 
y 3, pero con dificultades evidentes para operar con eslabones silábicos, así como para identificar palabras con premisas fonémicas determinadas.

Mientras que, la mayoría de los estudiantes que pertenecen a las instituciones educativas privadas alcanzan un desempeño con éxito en los primeros subtst del THM. Sin embargo, fracasan con respecto a las exigencias que plantean las sub pruebas 6 y 7 .

Tabla 4. Diferencia de los niveles de conciencia fonológica según tipo de instituciones educativas

\begin{tabular}{lcccc}
\hline $\begin{array}{l}\text { Niveles de } \\
\text { conciencia } \\
\text { fonológica }\end{array}$ & \multicolumn{2}{c}{$\begin{array}{c}\text { Institución educativa } \\
\text { pública }\end{array}$} & \multicolumn{2}{c}{ Institución educativa } \\
privada
\end{tabular}

Fuente: elaboración propia 


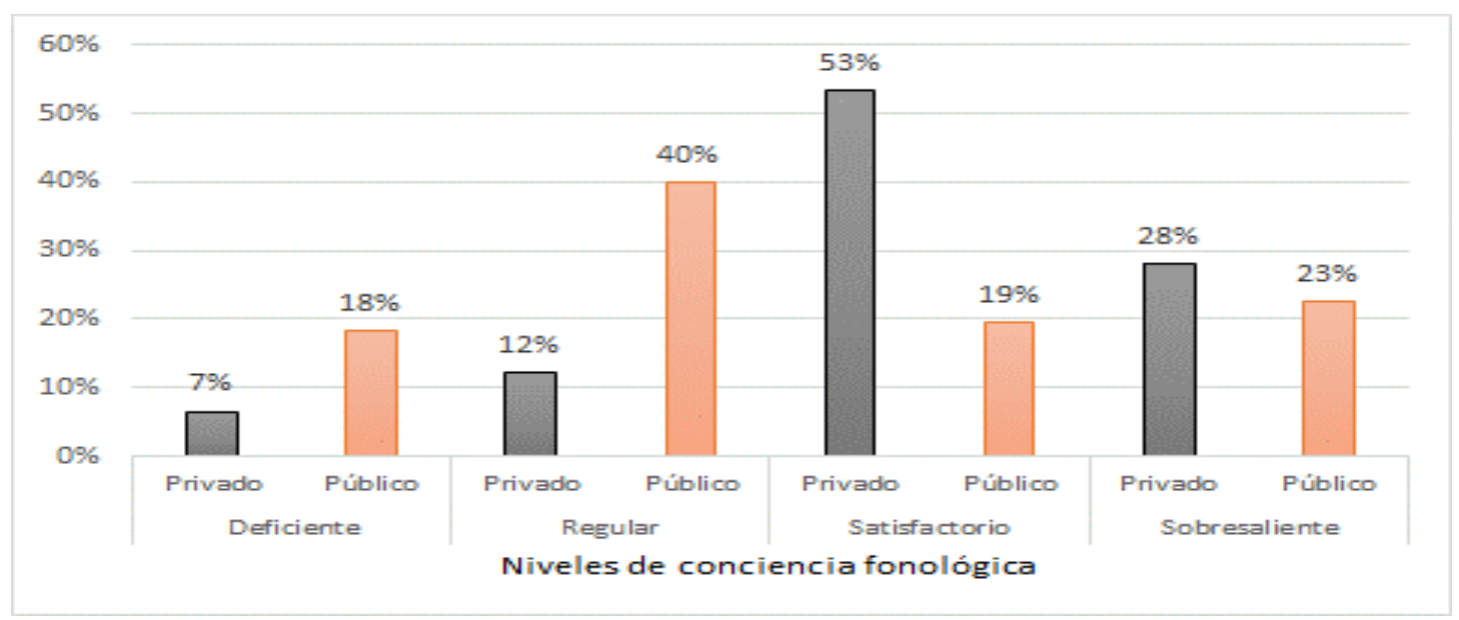

Figura 2. Distribución de porcentajes de los niveles de conciencia fonológica según tipo de instituciones educativas

\section{Niveles de conciencia fonológica según sexo}

En la tabla 5 y figura 3 se observa que; la mayoría de los estudiantes varones son capaces de desenvolverse con éxito en las sub pruebas 1 y 3 , pero con dificultades para operar con eslabones silábicos, así como para identificar palabras con premisas fonémicas determinadas.

Así también se observa que; las estudiantes mujeres alcanzan un desempeño con éxito en los primeros subtst del THM. Sin embargo, fracasan con respecto a las exigencias que plantean las pruebas 6 y 7 .

Tabla 5. Distribución de frecuencias de los niveles de conciencia fonológica según sexo

\begin{tabular}{lcccc}
\hline $\begin{array}{l}\text { Niveles de } \\
\text { conciencia } \\
\text { fonológica }\end{array}$ & $f$ & $\%$ & $f$ & $\%$ \\
\hline Deficiente & 23 & & 7 & $8 \%$ \\
\hline
\end{tabular}




\begin{tabular}{lcccc}
\hline & & $20 \%$ & & \\
Regular & 38 & $34 \%$ & 24 & $28 \%$ \\
Satisfactorio & 27 & $24 \%$ & 31 & $36 \%$ \\
Sobresaliente & 25 & $22 \%$ & 25 & $29 \%$ \\
Total & 113 & $100 \%$ & 87 & $100 \%$ \\
\hline
\end{tabular}

Fuente: elaboración propia

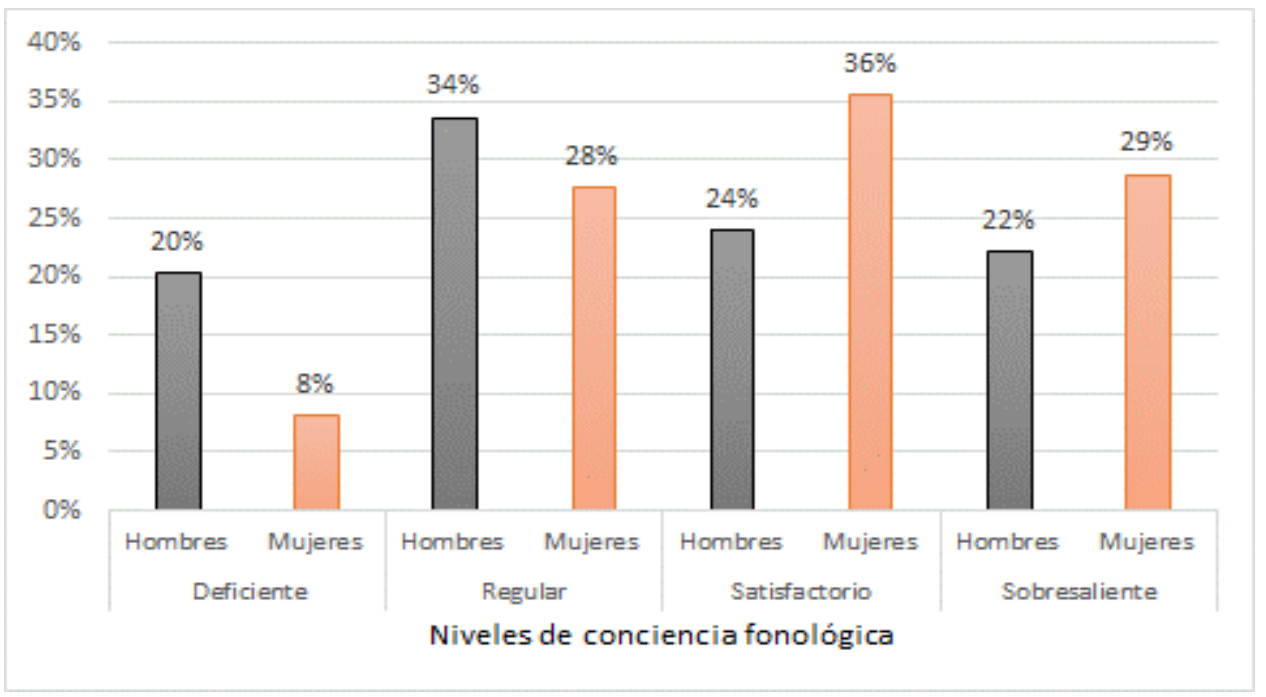

Figura 3. Distribución de porcentajes de los niveles de conciencia fonológica según sexo.

\section{Niveles de conciencia fonológica según edad}

En la tabla 6 y figura 4 se observa, que el mayor porcentaje de los estudiantes de 4 años de edad carecen de las habilidades fonológicas básicas, lo cual es fundamental en el acceso a la lectoescritura. Y los estudiantes de 5 años; son capaces de desenvolverse con éxito en las sub pruebas 1 y 3 , pero con dificultades manifiestas para operar con eslabones silábicos, así como para identificar palabras con premisas fonémicas determinadas. Mientras que, en los estudiantes de 6 años de edad, alcanzan con éxito en los primeros sub-test del THM. 
KOINONIA. Revista Arbitrada Interdisciplinaria de Ciencias de la Educación, Turismo, Ciencias Sociales y Económica, Ciencias del Agro y Mar y Ciencias Exactas y aplicadas. Año IV. Vol IV. Nº. Julio - Diciembre 2019. Hecho el depósito de Ley: FA2016000010

Tabla 6. Distribución de frecuencias de los niveles de conciencia fonológica según edad

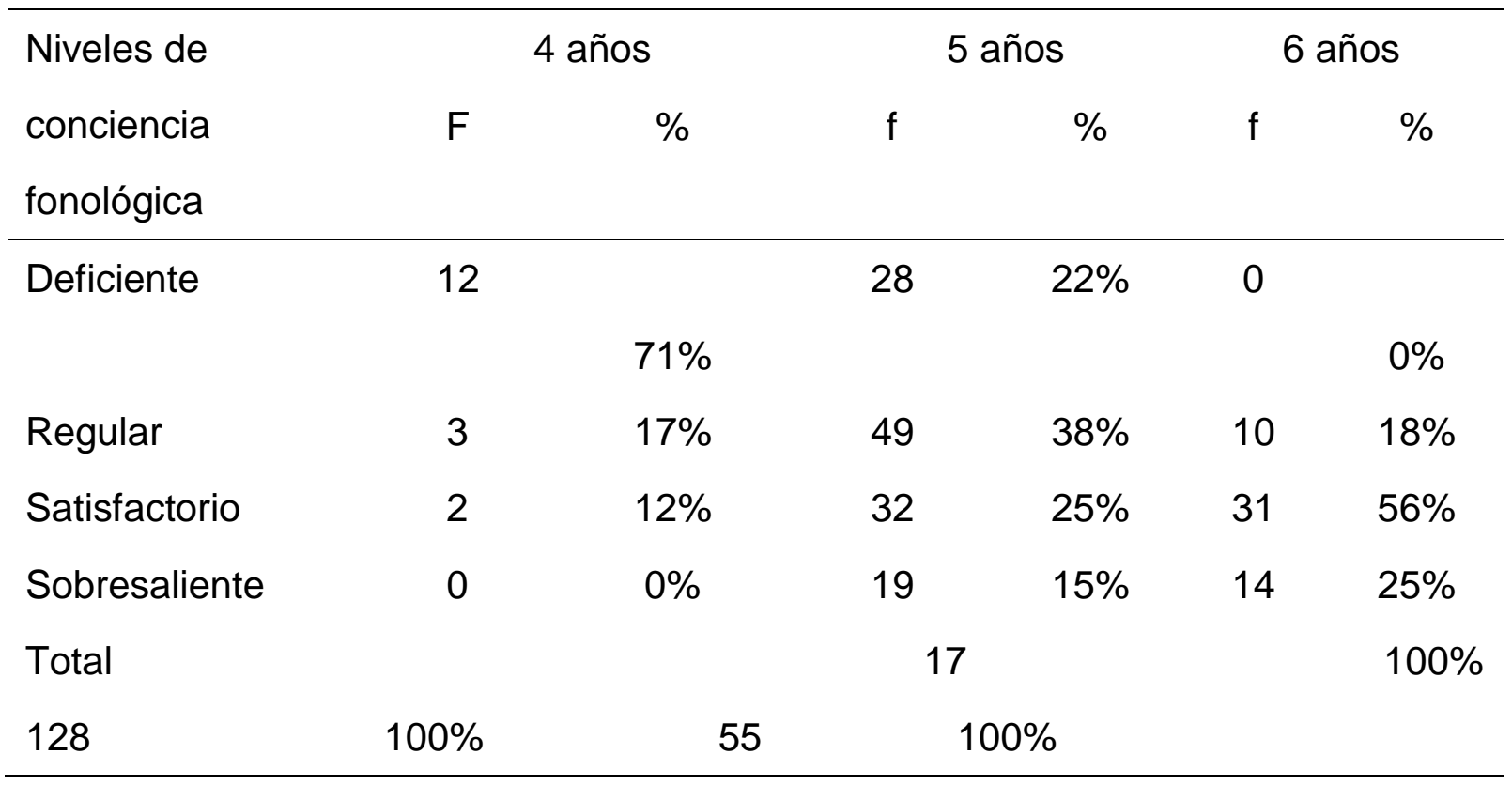

Fuente: elaboración propia

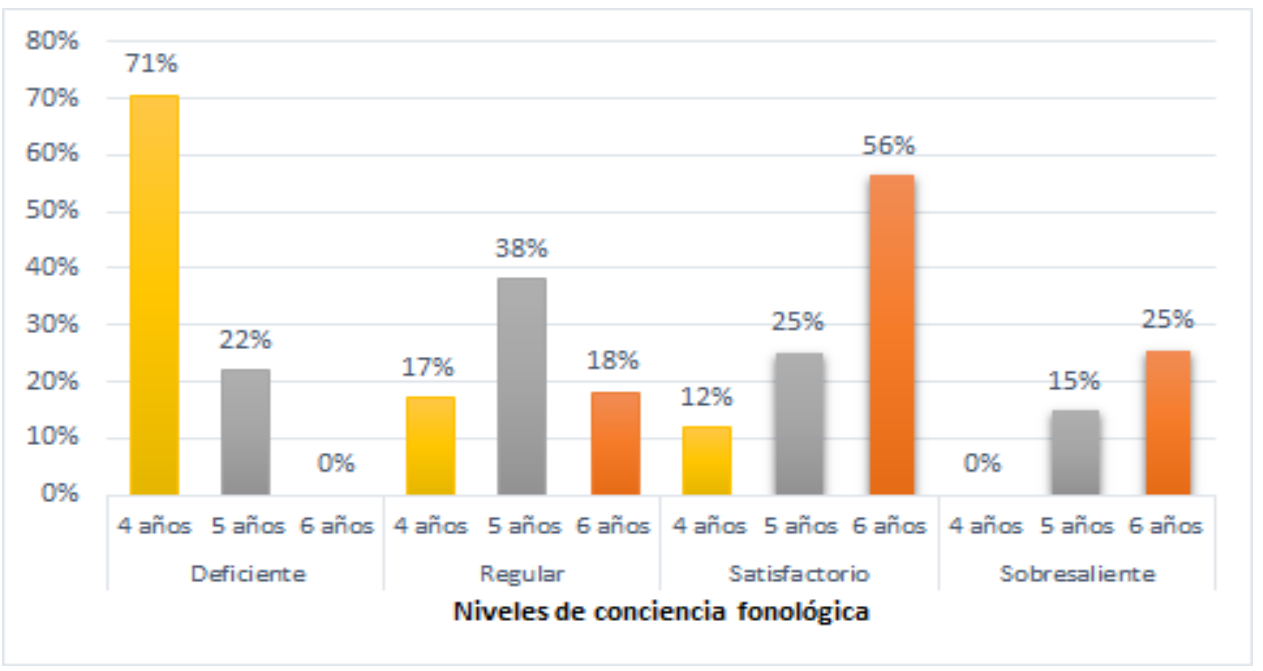

Figura 4. Distribución de porcentaje de los niveles de conciencia fonológica según edad

\section{Conciencia fonológica y sus dimensiones según tipo de familia}

En la tabla 7 se aprecian los promedios de la evaluación realizada sobre las dimensiones de conciencia fonológica en los estudiantes de instituciones educativas 
KOINONIA. Revista Arbitrada Interdisciplinaria de Ciencias de la Educación, Turismo, Ciencias Sociales y Económica, Ciencias del Agro y Mar y Ciencias Exactas y aplicadas. Año IV. Vol IV. Nº ${ }^{\circ}$. Julio - Diciembre 2019. Hecho el depósito de Ley: FA2016000010

públicas y privadas, según tipos de familia y los promedios son como sigue: En segmentación silábica $(17,75)$, el promedio más alto, lo tienen los estudiantes que provienen de familias tipo nuclear. En supresión silábica $(4,950)$ los estudiantes que provienen de familias tipo monoparental, en la dimensión Detección de rimas $(8,25)$ y en Adiciones Silábicas $(4,98)$ los estudiantes que provienen de familias tipo nuclear. En cuanto a la dimensión Aislamiento de Fonemas $(4,41)$ los estudiantes que provienen de familias tipo extensa y en Unión de Fonemas $(4,302)$ y Conteo de Fonemas $(5,524)$ el promedio más alto, lo obtuvieron los estudiantes que provienen de familias tipo extensa.

Tabla 7. Medias y desviación estándar de conciencia fonológica y sus dimensiones, según tipo de familia.

Monoparental

Nuclear

Extensa

\begin{tabular}{|c|c|c|c|c|c|c|}
\hline $\begin{array}{l}\text { Conciencia } \\
\text { fonológica }\end{array}$ & M & $S$ & $\mathrm{M}$ & $S$ & $M$ & $\mathrm{~S}$ \\
\hline $\begin{array}{l}\text { Segmentación } \\
\text { Silábica }\end{array}$ & 15.65 & 1,656 & 17.75 & 3,358 & 16.51 & 3,847 \\
\hline Supresión Silábica & 2.41 & 4,950 & 4.65 & 3,370 & 3.01 & 4,389 \\
\hline Detección de Rimas & 3.24 & 2,549 & 8.25 & 2,163 & 6.68 & 4,411 \\
\hline Adiciones Silábicas & 3.41 & 2,184 & 4.98 & 1,341 & 3.33 & 3,549 \\
\hline $\begin{array}{l}\text { Aislamiento de } \\
\text { Fonemas }\end{array}$ & 2.41 & 2,425 & 4.21 & 2,438 & 4.41 & 2,378 \\
\hline Unión de Fonemas & 1.59 & 2,959 & 3.49 & 1,110 & 3.13 & 4,302 \\
\hline Conteo de Fonemas & 2.76 & 4,764 & 3.97 & 3,125 & 3.98 & 5,524 \\
\hline
\end{tabular}

$\mathrm{n}=200$ 


\section{Conciencia fonológica y sus dimensiones según tipo de institución educativa}

En la tabla 8 se aprecian los promedios de la evaluación realizada sobre las dimensiones de la conciencia fonológica en los estudiantes, según tipos de institución educativa y los promedios más altos; lo tienen los estudiantes que provienen de colegios privados: en segmentación silábica $(18,11)$, supresión silábica $(4,21)$, detección de rimas $(8,64)$, Adiciones Silábicas $(4,21)$, Unión de Fonemas $(3,69)$ y Conteo de Fonemas $(2,78)$ y solamente en Aislamiento de Fonemas $(4,56)$ el promedio más alto; lo obtuvieron los estudiantes de instituciones públicas.

Tabla 8. Medias y desviación estándar de conciencia fonológica y sus dimensiones, según tipo de institución educativa

\begin{tabular}{lcccc}
\hline & \multicolumn{2}{c}{ Pública } & \multicolumn{2}{c}{ Particular } \\
\hline $\begin{array}{l}\text { Dimensiones Conciencia } \\
\text { fonológica }\end{array}$ & $\mathrm{M}$ & $\mathrm{S}$ & $\mathrm{M}$ & $\mathrm{S}$ \\
\hline Segmentación Silábica & 17,24 & 4,233 & 18,11 & 2,707 \\
Supresión Silábica & 3,11 & 4,461 & 4,21 & 5,200 \\
Detección de Rimas & 5,99 & 4,216 & 8,64 & 4,275 \\
Adiciones Silábicas & 3,19 & 3,794 & 4,21 & 3,229 \\
Aislamiento de Fonemas & 4,56 & 2,602 & 4,13 & 2,194 \\
Unión de Fonemas & 1,80 & 3,640 & 3,69 & 3,903 \\
Conteo de Fonemas & 1,27 & 5,505 & 2,78 & 5,053 \\
\hline
\end{tabular}

$\mathrm{n}=200$

\section{Conciencia fonológica y sus dimensiones según tipo género}

En la tabla 9 se aprecian los promedios de la evaluación realizada sobre las dimensiones de conciencia fonológica en los estudiantes, según género y los promedios más altos en: segmentación silábica $(17,82)$, Adiciones Silábicas $(3,71)$ y 
Aislamiento de Fonemas lo tienen los estudiantes del género masculino $(4,36)$, mientras que en: supresión silábica $(4,23)$, detección de rimas $(7,91)$, Unión de Fonemas $(3,61)$ y Conteo de Fonemas $(3,87)$ el promedio más alto lo obtuvieron, las estudiantes mujeres.

Tabla 9. Medias y desviación estándar de conciencia fonológica y sus dimensiones, según género

\begin{tabular}{lcccc}
\hline & \multicolumn{2}{c}{ Hombre } & \multicolumn{2}{c}{ Mujeres } \\
\hline $\begin{array}{l}\text { Dimensiones de conciencia } \\
\text { Fonológica }\end{array}$ & $\mathrm{M}$ & $\mathrm{S}$ & $\mathrm{M}$ & $\mathrm{S}$ \\
\hline Segmentación Silábica & 17,82 & 3,631 & 17,55 & 3,382 \\
Supresión Silábica & 3,29 & 4,692 & 4,23 & 5,114 \\
Detección de Rimas & 7,84 & 4,443 & 7,91 & 4,170 \\
Adiciones Silábicas & 3,71 & 3,504 & 3,64 & 3,576 \\
Aislamiento de Fonemas & 4,36 & 2,450 & 4,29 & 2,337 \\
Unión de Fonemas & 2,07 & 3,073 & 3,61 & 4,394 \\
Conteo de Fonemas & 3,16 & 5,133 & 3,87 & 5,530 \\
\hline
\end{tabular}

$n=200$

\section{Conciencia fonológica y sus dimensiones según edad}

En la tabla 10 se observa que los promedios más altos de la evaluación de las dimensiones de conciencia fonológica son del grupo de los estudiantes que tienen seis años de edad y es como sigue: en segmentación silábica $(18,15)$, supresión silábica $(4,45)$, Adiciones Silábicas $(5,49)$, Aislamiento de Fonemas $(5,34)$ y Conteo de Fonemas $(5,58)$ el promedio más alto lo tiene los estudiantes de 6 años. Mientras que los estudiantes que tienen cinco años de edad obtuvieron el puntaje más alto en: Detección de rimas $(7,89)$. 
KOINONIA. Revista Arbitrada Interdisciplinaria de Ciencias de la Educación, Turismo, Ciencias Sociales y Económica, Ciencias del Agro y Mar y Ciencias Exactas y aplicadas. Año IV. Vol IV. Nº ${ }^{\circ}$. Julio - Diciembre 2019. Hecho el depósito de Ley: FA2016000010

Tabla 10. Medias y desviación estándar de conciencia fonológica y sus dimensiones, según edad.

\begin{tabular}{lcccccc}
\hline & \multicolumn{2}{c}{4 años } & \multicolumn{2}{c}{5 años } & \multicolumn{2}{c}{6 años } \\
\hline \multicolumn{1}{c}{$\begin{array}{c}\text { Habilidades } \\
\text { metalingüísticas }\end{array}$} & M & s & M & s & M & s \\
\hline Segmentación Silábica & 13,18 & 3,283 & 16,76 & 3,390 & 18,15 & 3,912 \\
Supresión Silábica & 1,47 & 3,356 & 3,76 & 4,886 & 4,45 & 5,172 \\
Detección de Rimas & 3,82 & 4,433 & 7,89 & 4,206 & 7,53 & 4,562 \\
Adiciones Silábicas & 1,41 & 3,374 & 4,61 & 3,517 & 5,49 & 3,646 \\
Aislamiento de Fonemas & 2,94 & 2,015 & 4,99 & 2,424 & 5,34 & 2,427 \\
Unión de Fonemas & 1,18 & 1,741 & 3,66 & 3,616 & 4,41 & 4,467 \\
Conteo de Fonemas & 0,59 & 5,257 & 3,84 & 5,457 & 5,58 & 4,947 \\
\hline
\end{tabular}

$\mathrm{n}=200$

\section{CONSIDERACIONES FINALES}

El presente estudio asume la gran importancia de la conciencia fonológica en el aprendizaje de la lectura, pero su desarrollo está influenciado por la estimulación temprana en el contexto familiar, por lo tanto, debe ser contemplado en actividades de interacción y reflexión fonológica.

De este modo se asume que el desarrollo de ésta habilidad se asocia a las características de producción del lenguaje oral, observándose a partir de la articulación de sonidos, sílabas y palabras. Dado que al inicio los niños adquieren la habilidad separando las palabras en unidades silábicas siguiendo su orden articulatorio y pronto son capaces de aislar las sílabas iniciales o finales, comparándolas para averiguar si suenan igual o diferente. Es pues, sobre las bases de estas habilidades, proceso de reflexión sobre la fonología de las palabras que es 
posible iniciar el aprendizaje de la correspondencia entre las unidades silábicas y su forma escrita, de una manera comprensible para el aprendiz (Ferrer, 2007).

Dentro de los resultados obtenidos se encontró que existen diferencias significativas en las habilidades de conciencia fonológica según el tipo de familia en estudiantes de educación inicial de instituciones públicas y privadas de la UGEL 06. Los hallazgos descriptivos de este estudio dan cuenta que en el nivel deficiente se ubica, el mayor porcentaje de los estudiantes $44 \%$, los cuales provienen de una familia de tipo monoparental. En el nivel regular (37\%), se ubican los estudiantes que provienen de un tipo de familia extensiva y los niveles satisfactorio y sobresaliente $31 \%$ y $25 \%$, se ubican los estudiantes que provienen de una familia de tipo nuclear. $Y$ en el resultado inferencial se tiene que según la prueba Kruskal Wallis $(p=0,000<0,05)$ la conciencia fonológica, no es igual en los tres tipos de familia.

Estos resultados se fundamentan en lo expresados por Golombok (2006) "La mayoría de las familias monoparentales son el resultado de un matrimonio roto, y los hijos de padres divorciados son más propicios a tener problemas psicológicos y a tener más dificultades en su rendimiento escolar que los hijos de familias nucleares" (p.89). En el caso de las familia monoparentales, nucleares o extensas; se establecen diferencias entre estas interacciones de tal modo que los resultados obtenidos se observan diferencias en lo promedio, pero no son significativas en las habilidades metalingüísticas, según el tipo de familia, siendo las familias monoparentales las que obtiene un menor promedio en habilidades metalingüísticas y, por el contrario, las familias nucleares logran un mayor promedio.

Esto se podría deber a la existencia de diferencias lingüísticas en el estilo de interacción familiar, pues Bernstein (1971), sugiere que el uso del lenguaje en casa y los contextos de aprendizaje social proporcionan al niño o la niña, un abanico de experiencias lingüísticas que permite el desarrollo de la conciencia fonológica. En el caso de las familias nucleares la interacción se da en un modo más estable y profundo, en relación con las familias monoparentales o extensas. 
Así también lo demuestra los resultados de El Instituto de Ciencias para la Familia de la Universidad de Piura (2013), citado por Chuquimajo (2014), indica que la presencia de los padres en el hogar (dos padres, un padre o ninguno) es un factor fundamental para la consecución de algunos logros educativos como el nivel de comprensión lectora y la no repetición del grado escolar, medidos por la prueba Pisa 2009; además los niños que crecen con dos padres obtienen mejor puntaje en comprensión lectora, y tienen menos posibilidades de repetir el año, comparados con los niños de hogares monoparentales. La situación es más nociva aún para los niños que crecen sin padres, pues en el Perú se aproximan al 10\%. En lo referente a la familia extendida, esta puede conducir a sus miembros a una inestabilidad en la unidad familiar, existiendo varias figuras de autoridad (Lodo, 2007).

Gonzales (2005), añade que en el caso de las familias nucleares estas tareas de interacción se pueden alternar entre ambos padres en relación al niño, pero en el caso de las monoparentales no se podría realizar esta distribución en la interacción verbal, esto explicaría las diferencias en los promedios observados.

En este orden de ideas, se encontró que existen diferencias significativas en las habilidades de conciencia fonológica según tipo de instituciones educativas en estudiantes de educación inicial públicas y privadas de la UGEL 06. Los resultados descriptivos dan cuenta que en el nivel satisfactorio y sobresaliente se ubican, el mayor porcentaje de los estudiantes de las instituciones educativas privadas con el $43 \%$ y $32 \%$ respectivamente, y el $19 \%$ y $25 \%$, en los niveles regular y deficiente. Y en el resultado inferencial se tiene que según la prueba $U$ Mann Whitney $(p=0,000<$ 0,05) la conciencia fonológica, no es igual en los estudiantes según instituciones educativas.

Estos resultados coinciden con los encontrados por Aravena (2013), quien constató que los escolares provenientes de colegios particulares subvencionados alcanzaron un desempeño más favorable de conciencia fonológica, a diferencia de los escolares de colegios municipalizados. Tales resultados se sustentan en los expresados por 
Panca y Dioses (2000), quienes también confirmaron que, los alumnos procedentes de colegios privados superaron a los colegios públicos, tanto en la lectura de letras, silabas y palabras como en la lectura de textos y comprensión. Así también con los resultados de Balarezo (2007), afirmando que los alumnos procedentes de colegios privados muestran un mejor desempeño de conciencia fonológica, a diferencia de los alumnos de colegios públicos.

Asimismo, se encontró que existen diferencias significativas en las dimensiones de conciencia fonológica según sexo en estudiantes de educación inicial de instituciones públicas y privadas de la UGEL 06. Los resultados descriptivos dan cuenta que en el nivel sobresaliente se ubica, el mayor porcentaje de las estudiantes mujeres $36 \%$ y $29 \%$ en el nivel satisfactorio y sobresaliente. El $34 \%$ y $20 \%$, de los estudiantes varones, se ubican en los niveles regular y deficiente respectivamente. $Y$ en el resultado inferencial se tiene que según la prueba $U$ Mann Whitney. $(p=0,000<$ 0,05), lo cual indica que la conciencia fonológica, no es igual en los estudiantes según sexo.

Estos resultados coinciden con los encontrados por Panca y Dioses (2000), quienes también confirmaron que existían diferencias favorables al grupo de niñas en el rendimiento lector, tanto a nivel de los aspectos examinados, como de manera global. Además, discrepan con los resultados obtenidos por Céspedes (2010), quien encontró que no existen diferencias significativas en cuanto al sexo, de esta manera tanto niños como niñas poseen un rendimiento homogéneo en el Test de Habilidades Metalingüísticas, tanto en el puntaje global como al interior de los subtest. Por ello, cuando se debe de trata de implementar programas de desarrollo de la lectura según las necesidades de los niños, la discusión de las diferencias de sexo en el aprendizaje de la lectura aparece poco relevante. El rango de las diferencias en habilidades lectoras dentro de cada sexo es más grande que las diferencias entre un sexo y otro. Algunos niños están más preparados para iniciar el aprendizaje lector que algunas niñas y viceversa. 
Así también, concuerda con los resultados de Dioses García, Matalinares, Vuscano, Panca, Quiroz, Fernández y Castillo (2006) de un estudio sobre: Análisis psicolingüístico del desarrollo fonético fonológico de alumnos preescolares de Lima Metropolitana y publicado en la Revista de Investigación en Psicología, que dio como resultado que existen diferencias significativas en uso de la conciencia fonológico de rima y aliteración, según el nivel socioeconómico, sin embargo; las diferencias no eran significativas al considerarse las variables sexo y edad.

Por otro lado, se encontró que existen diferencias significativas en las habilidades de conciencia fonológica según edades en los estudiantes de educación inicial de instituciones públicas y privadas de la UGEL 06. Los resultados descriptivos dan cuenta que en el nivel deficiente se ubican, el mayor porcentaje de los estudiantes $59 \%$ de 4 años de edad, el $28 \%$ en el nivel regular $30 \%$ de los niños de 5 años y en el nivel satisfactorio y sobresaliente $55 \%$ y $25 \%$ se ubican los estudiantes de 6 años de edad. $Y$ en el resultado inferencial se tiene que según la prueba $U$ Mann Whitney $(p=$ $0,000<0,05)$, indicando que la conciencia fonológica, no es igual en los estudiantes según la edad.

Estos resultados coinciden con los expresados por algunos autores considerando que la aparición de la conciencia fonológica tiene lugar en torno a la edad de 4-5 años, mientras que otros la sitúan en 6-7 años, Bruce, (1964; citado en Jiménez y Ortiz, 1995). Estas discrepancias obedecen a los diferentes niveles de conciencia fonológica que los autores han considerado. Así, por ejemplo, los trabajos de Liberman y colaboradores (1977; citado en Jiménez y Ortiz, 1995), demuestran que contar sílabas es más fácil que contar fonemas. La conciencia de los fonemas es algo más difícil para los niños dado que los fonemas aparecen co-articulados en las palabras. Así también, con lo señalado por Negro y Traverso (2011), quienes afirman que la conciencia fonológica evoluciona con el tiempo, a la edad de cinco años aproximadamente se puede percibir en los niños el desarrollo del lenguaje hablado, en definitiva, el momento de máximo desarrollo del lenguaje, es cuando la mayoría de 
los niños, han adquirido el conocimiento suficiente de grafemas, sílabas y palabras; factores relacionados con el aprendizaje de la lectura y escritura.

En relación a la estructura familiar, en estudiantes de Educación Inicial de Instituciones Públicas y Privadas de la UGEL 06, los resultados dan cuenta que el estadístico tiene asociado niveles crítico bilaterales menores a 0,05, en el caso de segmentación silábica, detección de rimas y conteo de fonemas, por lo tanto para estos casos se puede rechazar la hipótesis de igualdad de medias y concluir que las medias en estas dimensiones de las habilidades metalingüísticas se presentan diferencias significativas.

Resultados que coinciden con los hallados por Céspedes (2010), quien señala que la conciencia silábica es una de las habilidades de más fácil reconocimiento para niños y adultos analfabetos, mientras que la conciencia de la rima es el conocimiento de nivel más básico y está fuertemente relacionada con la habilidad posterior en lectura.

Del mismo modo, se encontró que las tareas de segmentación silábica son más fáciles que las tareas de rimas y de reconocimiento fonémico, coincidiendo con la investigación realizada por Jiménez y Ortiz (1995) citados por Céspedes (2010) quienes realizaron una investigación con niños pre lectores en España, donde los niños tenían un mejor rendimiento en tareas relacionadas con separar palabras en sílabas y presentaron una mayor dificultad en ejercicios que demandaban una conciencia fonémica. Esto se debe a que la sílaba es la unidad básica del habla en el castellano, mientras que la conciencia de los fonemas es algo más difícil para los niños pues aparecen co articulados en las palabras (Jiménez y Ortiz, 1995).

Asimismo, se encontró que existen diferencias significativas en todas las dimensiones de conciencia fonológica según tipo de instituciones educativas, en estudiantes de Educación Inicial de Instituciones Públicas y Privadas de la UGEL 06. Los resultados indican que existen diferencias significativas en las habilidades metalingüísticas segmentación silábica, detección de rimas y conteo de fonemas, según el tipo de institución educativa. Las diferencias entre instituciones se podrían 
observar debido a los diferentes modelos de enseñanza y estimulación de la lectoescritura.

Estos resultados coinciden con lo señalado por Panca y Dioses (2000), al evaluar las habilidades metalingüísticas y rendimiento lector en alumnos de primer grado de educación primaria de colegios públicos y privados, los resultados mostraron que los alumnos de colegios públicos evidenciaban diferencias significativas en sus habilidades metalingüísticas y rendimiento lector con respecto a los alumnos de colegios privados, y en específico en segmentar palabras en silabas y la capacidad de identificar los fonemas.

Además, Dioses et al. (2006), demostró diferencias significativas en uso de la conciencia fonológico, según el nivel socioeconómico, dichos resultados coinciden con lo observado por Velarde y Canales (2008), al comparar los rendimientos según la gestión educativa, se encontró que el rendimiento de los niños y niñas de las instituciones educativas estatales más pobres era significativamente inferior al rendimiento de los niños y niñas, igualmente pobres, que estudiaban en la institución educativa parroquial. Esto indicaría que no es solo las diferencias se presenta a favor de escuelas privadas o parroquial en relación con las instituciones educativas públicas, sino que las diferencias se deben observar en los programas y métodos de enseñanza de la lectura.

Respecto al género, se encontró que no existen diferencias significativas en todas las dimensiones de conciencia fonológica. Los resultados indican diferencias significativas en las habilidades metalingüísticas segmentación silábica, detección de rimas y unión de fonemas, estos resultados coinciden con lo encontrado por Dioses et al. (2006), encontrando diferencias significativas al considerarse las variables de sexo, aunque los niños superan no significativamente a las niñas.

Así también con Matalinares y Díaz (2007), quienes establecieron la relación existente entre las habilidades metalingüísticas y la comprensión en niños y niñas de primer grado de las ciudades de Lima y Huancayo, pero al comparar las muestras no 
se encontraron diferencias significativas en las habilidades metalingüísticas, ni en comprensión, cuando se comparó el puntaje general en habilidades metalingüísticas y comprensión entre hombres y mujeres no se hallaron diferencias significativas.

Finalmente, los resultados indican que existen diferencias significativas en las habilidades metalingüísticas a favor del grupo de 5 y 6 años de edad. Estos resultados concuerdan con los encontrados Coloma, Cobarrubias y De Barbieri (2007), cuyos resultados obtenidos indican que los preescolares de 5 años evidencian un rendimiento significativamente mayor al grupo de niños de 4 años en tareas de conciencia fonológica. Lo anterior, permite concluir que existe un incremento de las habilidades metalingüísticas relacionadas con la sílaba al comparar preescolares de 4 y 5 años.

\section{REFERENCIAS CONSULTADAS}

1. Balarezo, P. (2007). Nivel de conciencia fonológica en los niños y niñas de primer grado de Instituciones Educativas Públicas y Privadas del distrito de Pueblo Libre. Tesis de Maestría en Educación. Pontificia Universidad Católica del Perú.

2. Carrasco, S. (2009) Metodología de investigación científica: Pautas metodológicas para diseñar y elaborar el proyecto de investigación. Lima: Marcos.

3. Céspedes, A. (2010). Comparación de la conciencia fonológica en niños y niñas de 5 años de la IE 84 del Callao. Tesis. Lima Universidad San Ignacio de Loyola.

4. Coloma, C. e Infante, K. (2011). Habilidades Metalingüísticas. Apuntes de clases Psicolingüística para III de Fonoaudiología.

5. Dioses, A. (2006). Analisis Psicolinguistico del desarrollo fonológico. en alumnos pre escolares de Lima Metropolitana

6. Dioses, A. y Panca, N. (2000). Habilidades metalingüísticas y rendimiento lector en alumnos del primer grado de primaria de colegios públicos y privados de San Juan de Lurigancho. 
7. Gómez, P., Valero, J., Buandes, R y Pérez, A. (1995). Manual de Test de Habilidades Metalingüísticas. Madrid: Instituto de Orientación Psicológica.

8. Jiménez y Ortiz M. (1995) Conciencia Fonológica y Aprendizaje a la Lectura, teoría, evaluación e interpretación, La Laguna: Editorial Síntesis.

9. Jiménez, L. Y. (2012). Comprensión lectora y rendimiento académico en comunicación de alumnos del segundo grado de una institución educativa de Ventanilla.

10. Mata C. y Macassi S. (1997) Cómo elaborar muestras para los sondeos de audiencias. Cuadernos de investigación No 5. ALER, Quito.

11. Matalinares, M. y Díaz. A. (2007). Habilidades metalingüísticas y la comprensión en niños de primer grado de las ciudades de Lima y Huancayo. Universidad César Vallejo-Lima y Universidad Nacional Mayor de San Marcos. Revista de Psicología Vol. 9. Trujillo.

12. MINEDU (2014). Resultados de la evaluación censal de estudiantes. Lima.

13. Negro y Traverso (2011). Relación entre la conciencia fonológica y la lectura inicial en alumnos de primer grado de educación primaria de los centros educativos "Héroes del Cenepa" y "Viña Alta" de La Molina - Lima. Tesis. Lima: Pontificia Universidad Católica del Perú.

14. Panca, N. (2004). Relación entre habilidades metalingüísticas y el rendimiento lector en un grupo de alumnos de condición socioeconómico baja que cursan el primer grado de Educación Primaria. Universidad Pontificia Católica del Perú.

15. Velarde, E. (2001). Relación de la conciencia fonológica y el nivel de decodificación y comprensión lectora en niños de 8 años del $3^{\circ}$ grado de primaria de dos niveles socioeconómicos del Cercado del Callao. Lima: Tesis para optar el título de Licenciatura en Psicología. Universidad Nacional Mayor de San Marcos.

16. Velarde, E. y Canales, R. (2008). La Lectura en el Perú: Drama y esperanza. Lima: Universitaria.

17. Velarde, E (2009) Habilidades Pre Lectoras. Universidad San Ignacio De Loyola Facultad de Educación Programa Académico de Maestría en Educación - Pame Callao. 
18. Velarde, E.; Canales, R.; Meléndez, M. y Lingán, S. (2010) Enfoque cognitivo y psicolingüístico de la lectura: Diseño y validación de una prueba de habilidades prelectoras (THP) en niños y niñas de la provincia constitucional del Callao, Perú. Revista IIPS, Vol. 13, № 1, pp. 53 - 68.

\section{REFERENCES CONSULTED}

1. Balarezo, P. (2007). Level of phonological awareness in the children of first grade of Public and Private Educational Institutions of the district of Pueblo Libre. Master's Thesis in Education. Pontifical Catholic University of Peru.

2. Carrasco, S. (2009) Scientific research methodology: Methodological guidelines to design and develop the research project. Lima: Marcos.

3. Céspedes, A. (2010). Comparison of phonological awareness in children of 5 years of El 84 of Callao. Thesis. Lima University San Ignacio de Loyola.

4. Coloma, C. and Infante, K. (2011). Metalinguistic skills. Notes of classes Psycholinguistics for III of Speech Therapy.

5. Gods, A. (2006). Psycholinguistic analysis of phonological development. in pre-school students of Metropolitan Lima

6. Gods, A. and Panca, N. (2000). Metalinguistic skills and reading performance in first grade students of public and private schools of San Juan de Lurigancho.

7. Gómez, P., Valero, J., Buandes, R and Pérez, A. (1995). Metalinguistic Skills Test Manual. Madrid: Institute of Psychological Guidance.

8. Jiménez and Ortiz M. (1995) Phonological Awareness and Reading Learning, theory, evaluation and interpretation, La Laguna: Editorial Síntesis.

9. Jiménez, L. Y. (2012). Reading comprehension and academic performance in communication of students of the second grade of a educational institution of Ventanilla.

10. Mata C. and Macassi S. (1997) How to prepare samples for audience surveys. Cuadernos de investigación No 5. ALER, Quito. 
11. Matalinares, M. and Díaz. A. (2007). Meta-linguistic skills and understanding in first-grade children in the cities of Lima and Huancayo. César Vallejo-Lima University and National University of San Marcos. Journal of Psychology Vol. 9. Trujillo.

12. MINEDU (2014). Results of the census evaluation of students. Lime.

13. Negro and Traverso (2011). Relationship between phonological awareness and initial reading in primary school students of the "Héroes del Cenepa" and "Viña Alta" educational centers in La Molina - Lima. Thesis. Lima: Pontifical Catholic University of Peru.

14. Panca, N. (2004). Relationship between metalinguistic skills and reading performance in a group of students of low socioeconomic status who attend the first grade of Primary Education. Pontifical Catholic University of Peru.

15. Velarde, E. (2001). Relation of the phonological awareness and the level of decoding and reading comprehension in children of 8 years of the 3rd grade of primary of two socioeconomic levels of the Cercado del Callao. Lima: Thesis to choose the Bachelor's degree in Psychology. National University of San Marcos.

16. Velarde, E. and Canales, R. (2008). The Reading in Peru: Drama and hope. Lima: University.

17. Velarde, E (2009) Pre-reading Skills. San Ignacio De Loyola University Faculty of Education Academic Program of Master of Education - Pame Callao.

18. Velarde, E.; Canales, R .; Meléndez, M. and Lingán, S. (2010) Cognitive and psycholinguistic approach to reading: Design and validation of a pre-literacy test (THP) in boys and girls from the constitutional province of Callao, Peru. IIPS Magazine, Vol. 13, No. 1, pp. 53-68. 